\title{
Suplementos ergogénicos: la evidencia más allá de una moda
}

\author{
Ergogenic supplements: evidence beyond trends
}

\author{
Miquel Martorell ${ }^{1,2}$, Karina Ramírez-Alarcón' , Ana María Labraña', Fabián Lanuza ${ }^{3,4}$, María Adela Martínez-Sanguinetti \\ Ana María Leiva-Ordóñez ${ }^{6}$, Claudia Troncoso-Pantoja ${ }^{7}$, Marcelo Villagrán ${ }^{8}$, Lorena Mardones ${ }^{8}$, Carlos Celis-Morales ${ }^{9,10,11}$, \\ Fanny Petermann-Rocha ${ }^{9,12}$
}

\section{Resumen}

A pesar de que son muchos los suplementos deportivos que se etiquetan como ergogénicos, pocos son los que realmente demuestran su efectividad. Si bien estos suplementos están destinados principalmente para deportistas de élite, su uso se ha masificado en la población general, esto debido a la falta de regulación en uso y venta, así como por los potenciales beneficios que podrían otorgar. Frente a estas limitantes ¿cuál es la efectividad de estos productos más allá de la moda? En esta comunicación breve se revisa la evidencia respecto al efecto y la efectividad de 11 suplementos y plantas utilizados como ayuda ergogénica. A pesar de los múltiples beneficios en los suplementos ergogénicos identificados, es necesario tener precaución al momento de generalizar los resultados obtenidos por algunos ergogénicos, ya que su efecto podría ser secundario y/o estar condicionado por otros factores. Es necesario mayores estudios clínicos que permitan asociar una relación causa-efecto en el rendimiento deportivo y la población general.

Palabras clave: sustancias ergogénicas; suplementos dietéticos; dieta; suplementos de hierbas; deporte; efecto ergogénico.

\begin{abstract}
Although many sports supplements have been identified as ergogenic, few have demonstrated their effectiveness. They were originally created for high-performance athletes, but their consumption has incremented in the general population. Lack of regulation to sell and use these products is one of the main problems in our society. In this context, what is the effectiveness of ergogenic supplements beyond the trends? In this short communication, 11 ergogenic supplements and plants will be discussed regarding their effect and efficacy. However, considering that their effect could be a side effect of other factors, findings should be analyzed with caution. Further clinical trials are needed to estimate the causal effect in sports performance and the general population.
\end{abstract}

Keywords: ergogenic substances; dietary supplements; diet; herbal supplement; sports.

(1) Departamento de Nutrición y Dietética, Facultad de Farmacia, Universidad de Concepción, Concepción, Chile.

(2) Centro de Vida Saludable, Universidad de Concepción, Concepción, Chile

(3) Biomarkers and Nutrimetabolomics Laboratory, Department of Nutrition, Food Sciences and Gastronomy, Food Technology Reference Net (XaRTA), Nutrition and Food Safety Research Institute (INSA), Faculty of Pharmacy and Food Sciences, University of Barcelona, Barcelona, Spain.

(4) Centro de Epidemiología Cardiovascular y Nutricional (EPICYN), Facultad de Medicina, Universidad de La Frontera, Temuco, Chile.

(5) Instituto de Farmacia, Facultad de Ciencias, Universidad Austral de Chile, Valdivia, Chile.

(6) Instituto de Anatomía, Histología y Patología, Facultad de Medicina, Universidad Austral de Chile, Valdivia, Chile.

(7) Departamento de Salud Pública. Facultad de Medicina, CIEDE-UCSC, Universidad Católica de la Santísima Concepción, Concepción, Chile

(8) Departamento de Ciencias Básicas. Facultad de Medicina. Universidad Católica de la Santísima Concepción, Concepción, Chile.

(9) British Heart Foundation Glasgow Cardiovascular Research Centre, Institute of Cardiovascular and Medical Science, University of Glasgow, Glasgow.

(10) Laboratorio de Rendimiento Humano, Grupo de Estudio en Educación, Actividad Física y Salud (GEEAFyS), Universidad Católica del Maule, Chile

(11) Centro de Investigaciones en Fisiología del Ejercicio (CIFE), Universidad Mayor, Chile

(12) Institute of Health and Wellbeing, University of Glasgow, Glasgow, UK. G12 $8 R Z$

Autor de Correspondencia: f.petermann-rocha.1@research.gla.ac.uk 


\section{Introducción}

El uso de suplementos deportivos ha aumentado ampliamente, utilizándose para la obtención de un mejor rendimiento en deportistas de élite o como una moda mal orientada para alcanzar objetivos en un corto plazo. En el caso de los deportistas de élite, se estima que entre un 40 y un $100 \%$ de ellos utiliza este tipo de productos para obtener un mejor estado de salud, un mejor rendimiento deportivo o simplemente maximizarlo (Garthe \& Maughan, 2018). No obstante, su uso también ha aumentado en población general. En Estados Unidos, más de un 71\% de los adultos declaró consumir algún tipo de suplemento en el año 2016 ya sea por razones de salud, obtención de energía, bienestar o complementar nutrientes en la dieta (Garthe \& Maughan, 2018). En Reino Unido, el Servicio Nacional de Salud (NHS) ha reportado un aumento considerable en el consumo de estos productos, principalmente con el objetivo de perder peso corporal y desarrollar masa muscular (NHS, 2018). En Chile, Jorquera-Aguilera et al. (2016) identificaron que los hombres consumen una mayor proporción de este tipo de suplementos que las mujeres con el objetivo de aumentar su masa muscular (34,9\% vs 20,0\%). En el caso de las mujeres, estas los suelen consumir para disminuir la grasa corporal, sin recibir una asesoría profesional previa sobre su uso (Jorquera Aguilera et al., 2016). De igual manera, Gallardo et al. (2015) determinaron que un $22 \%$ de los usuarios que asistían a gimnasios en el sur de Chile consumían algún tipo de suplemento deportivo para aumentar la masa muscular o mejorar el rendimiento físico. Sin embargo, ¿cómo se definen los suplementos ergogénicos? El concepto viene del griego "ergon" trabajo y "gennan" producción y hace referencia a cualquier sustancia, método o fármaco que contribuya a mejorar el rendimiento o trabajo físico de un deportista. Uno de los principales problemas hasta ahora es la falta de regulación del uso y venta de estos productos, los cuales no solamente son consumidos por deportistas de alto rendimiento, sino que también por un gran número de personas que frecuenta de manera activa los gimnasios o quienes buscan una solución rápida y efectiva de su condición y apariencia física antes de iniciar el verano. En la gran mayoría de los casos, las dosis efectivas de estos suplementos se pueden lograr aumentando ligeramente el consumo de alimentos y bebidas de uso común (por ejemplo, cafeína a través de café y nitrato a través de vegetales de hoja verde o raíces) y en otros casos se requiere la ingesta de un suplemento específico (creatina, bicarbonato de sodio y $\beta$-alanina). Sin embargo, es importante tener precaución al momento de generalizar los resultados obtenidos por este tipo de productos, ya que su efecto podría estar condicionado por otros factores, tales como una dieta hipocalórica o el inicio de un nuevo programa de ejercicio físico de forma paralela y el estado nutricional de la persona. En este contexto, ¿cuál es la efectividad y el efecto de estos productos más allá de la moda? El objetivo de esta comunicación breve es identificar la efectividad y el efecto de los suplementos y plantas más utilizados como ayudas ergogénicas que son de fácil acceso a la población general y con evidencia científica reportada.

\section{Métodos}

Esta comunicación breve se realizó consultando las bases de datos PubMed, Web of Science y Scielo (como motor de búsqueda) para obtener los artículos más actualizados sobre el tema en revisión (suplementos ergogénicos contrastados en humanos). La estrategia de búsqueda incluyó el uso de las siguientes palabras clave: "ergogenic aids", "supplementation", "performance", "ayudas ergogénicas" $y$ "suplementación deportiva". Los autores examinaron cuidadosamente los artículos para identificar sus fortalezas y debilidades, y seleccionaron los más útiles para el propósito de la revisión, priorizando los artículos publicados en los últimos 10 años; incluyendo algunos artículos publicados en años anteriores a los descritos, cuando existía escasa evidencia del suplemento buscado. Se consideraron solo artículos en inglés y español con texto completo. Se analizaron 11 suplementos que se detallan a continuación.

\section{Suplementos ergogénicos: resultados de búsqueda}

\section{Cafeína}

Su función como antagonista del receptor de adenosina es el mecanismo al que principalmente se le atribuye su efecto ergogénico, aunque también se proponen mecanismos que implican el aumento de secreción de endorfinas y la mejora de la función neuromuscular (Southward et al., 2018). Producto de su modulación hormonal, la cafeína favorece un estado de alerta y vigilancia, y una mayor movilización intracelular de calcio y ácidos grasos libres en músculo (Southward et al., 2018). Además, la cafeína mejora la capacidad de resistencia al ejercicio prolongando el tiempo de aparición de la fatiga durante el ejercicio (Guest et al., 2021). La resistencia aeróbica parece ser la forma de ejercicio con los beneficios más consistentes del uso de cafeína, aunque la magnitud de sus efectos difiere entre individuos (Guest et al., 2021). Dosis moderadas de cafeína (3-6 mg/kg peso corporal) consumidas 60 minutos antes del ejercicio parecen tener los efectos más efectivos, existiendo numerosos protocolos que han demostrado su efectividad (Southward et al., 2018; Guest et al., 2021 ). Las dosis mínimas efectivas no están claras, pero parecen situarse cerca de $2 \mathrm{mg} / \mathrm{kg}$ peso corporal. Por otro lado, dosis muy elevadas $(9 \mathrm{mg} / \mathrm{kg}$ peso corporal) se han asociado a efectos secundarios sin ser necesarias para provocar un efecto ergogénico (Guest et al., 2021). En síntesis, la efectividad de la cafeína como suplemento se evidenciaría en algunos deportes, en especial, los que buscan 
aumentar levemente la resistencia aeróbica; ya que podría provocar un incremento modesto, pero relevante, en competiciones donde los atletas están separados por márgenes muy pequeños.

\section{Nitrato}

El nitrato dietético $\left(\mathrm{NO}_{3}{ }^{-}\right)$mejora la cinética del consumo de oxígeno $\left(\mathrm{VO}_{2}\right)$ durante ejercicios prolongados sub-máximos, mediante el aumento en la biodisponibilidad de óxido nítrico (NO), a través de la vía $\mathrm{NO}_{3}^{-}$- nitrito $\left(\mathrm{NO}_{2}^{-}\right)$- NO catalizada por bacterias presentes en la mucosa oral y el sistema digestivo. El NO es conocido como un potente vasodilatador que favorece la irrigación sanguínea hacia los músculos, mejorando el aporte de sustratos energéticos para la respiración aeróbica como oxígeno, ácidos grasos, glucosa; así como la remoción de intermediarios y desechos metabólicos (Betteridge et al., 2016). Además, el rol ergogénico de la producción de NO parece estar relacionado con el tipo de fibra muscular, siendo las fibras tipo II (glucolíticas de contracción rápida) las que se verían más beneficiadas (Hlinský et al., 2020). Los vegetales de hoja verde y de raíz como espinaca, rúcula, rábano, remolacha y apio, son fuentes dietarias importantes de $\mathrm{NO}_{3}^{-}$. Estos vegetales contienen aproximadamente $250 \mathrm{mg}$ de $\mathrm{NO}_{3}{ }^{-}$por $100 \mathrm{~g}$ de peso fresco. Se ha estimado que su ingesta típica varía entre 31 y $185 \mathrm{mg} \mathrm{NO}_{3}^{-}$/ día en Europa y entre 40 y 100 mg NO ${ }_{3}^{-}$/día en Estados Unidos (Hord et al., 2009). La mayoría de los estudios de intervención que han evaluado el impacto del $\mathrm{NO}_{3}{ }^{-}$sobre el rendimiento deportivo, se han realizado con jugo de remolacha. Mejoras en el tiempo hasta llegar al agotamiento, se han evidenciado con dosis de 300-600 mg de $\mathrm{NO}_{3}$-, en estudios con una sola ingesta aguda y hasta con 15 días de ingestión regular (Van De Walle \& Vukovich, 2018). El efecto ergogénico se ha reportado mayormente en hombres jóvenes, sanos y que realizan actividad física recreativa, aunque el efecto de la suplementación se considera leve (Senefeld et al., 2020). Los efectos ergogénicos del $\mathrm{NO}_{3}^{-}$se han reportado mayormente en protocolos de suplementación crónica y no en suplementación aguda (Hlinský et al., 2020).

\section{Creatina}

La creatina es una molécula sintetizada endógenamente a partir de los aminoácidos arginina, glicina y metionina. Como suplemento, su forma más común de presentación es como monohidrato de creatina y, tras un protocolo de carga y/o mantención (por ej. 0,3 $\mathrm{g} / \mathrm{kg}$ peso corporal 4 veces al día por 5-7 días), la creatina intramuscular puede incrementarse hasta un 20-30\% (Kreider et al., 2017). Esta mayor biodisponibilidad celular permite un incremento en la resíntesis de fosfocreatina, contribuyendo a mantener un pool adicional de fosfatos de alta energía que permitirán aportar energía (ATP) durante los primeros segundos de contracción muscular. De esta manera, la creatina mejora la capacidad de realizar episodios repetidos de ejercicios de alta intensidad y de corta duración. La suplementación con creatina a corto plazo se ha asociado a una mayor retención de agua intracelular, provocando un aumento de peso corporal y pudiendo afectar negativamente el rendimiento en deportes peso-dependientes (Kreider et al., 2017). Sin embargo, la suplementación a largo plazo no se ha asociado a retención de agua, ni intra ni extracelular (Antonio et al., 2021).

\section{Bicarbonato de sodio $\left(\mathrm{NaHCO}_{3}\right)$}

El bicarbonato posee un rol importante como tampón fisiológico debido a que su suplementación provoca un aumento de gradiente entre el entorno intra y extracelular, lo que favorece un mayor flujo de salida intramuscular de protones y lactato durante ejercicios de alta intensidad. Esto provoca un retraso de los efectos perjudiciales de la acidificación intramuscular que afecta a la homeostasis del ion calcio $\left(\mathrm{Ca}^{2+}\right)$, la contracción muscular y la homeostasis energética (Hadzic et al., 2019). Ingestas de 0,2-0,4 $\mathrm{g} \mathrm{NaHCO}_{3} / \mathrm{kg}$ peso corporal provocan un peak máximo de $\mathrm{HCO}_{3}{ }^{-}$a los 60-150 minutos post-ingesta (Siegler et al., 2012). La suplementación con $\mathrm{NaHCO}_{3}$ mejora la resistencia muscular de grupos de músculos pequeños y grandes, aumentando la fuerza muscular (Grgic et al., 2020). La duración del ejercicio parece fundamental para observar el efecto ergogénico de $\mathrm{NaHCO}_{3^{\prime}}$, siendo en ejercicios de duración menor a los 4 minutos donde más se han reportado efectos positivos, aunque existen inconsistencias (Hadzic et al., 2019). Entre los efectos adversos de la administración de este suplemento, se reporta malestar gastrointestinal (distención abdominal, náuseas, y/o diarrea), condiciones que afectan negativamente cualquier mejora en el rendimiento deportivo (Santesteban Moriones \& Ibáñez Santos, 2017). Para una menor probabilidad de malestar gastrointestinal, se sugiere ingerir el suplemento $\left(0,3 \mathrm{~g} \mathrm{NaHCO}_{3} /\right.$ $\mathrm{kg}$ peso corporal) con una comida rica en carbohidratos y $7 \mathrm{~mL}$ de agua/kg peso corporal entre 2 y 2,5 horas antes de la práctica deportiva (Carr et al., 2011).

\section{$\beta$-alanina}

La $\beta$-alanina es un aminoácido no esencial y su suplementación incrementa los niveles de carnosina dentro del músculo esquelético (Huerta Ojeda et al., 2020). La carnosina es una sustancia tampón que se sintetiza por acción de la carnosina sintetasa al unir $\beta$-alanina y L-histidina. Al igual que el bicarbonato de sodio, la carnosina incrementa la capacidad de amortiguación de protones producidos durante la contracción muscular, retardando la fatiga producida por acidificación muscular (Huerta Ojeda et al., 2020). La suplementación diaria con aproximadamente $65 \mathrm{mg}$ de $\beta$-alanina/kg de peso corporal por un periodo mínimo de 2 a 4 semanas, incrementa 
el contenido de carnosina intramuscular y mejora la tolerancia a entrenamientos máximos de 30 segundos a 10 minutos (Saunders et al., 2017). En las zonas de transición aeróbica-anaeróbica, la suplementación con $\beta$-alanina ha mostrado pequeños efectos sobre el rendimiento deportivo (Huerta Ojeda et al., 2020). En individuos bien entrenados, con una capacidad de amortiguación del pH intramuscular ya mejorada, la suplementación con $\beta$-alanina no es tan efectiva como en individuos menos entrenados. El principal efecto adverso observado con la suplementación de $\beta$-alanina, es la parestesia, la cual podría influir en el rendimiento del deportista (Santesteban Moriones \& Ibáñez Santos, 2017).

\section{Ginseng (Panax ginseng C.A.Mey.)}

Las raíces del gingseng son muy populares como suplemento a nivel mundial (Sellami et al., 2018; Williams, 2006). Además de sus efectos sobre el estrés, la mejoría del sistema inmune y la presión sanguínea; a nivel deportivo (dosis $\leq 200 \mathrm{mg} /$ día por 9 semanas) ha demostrado efectividad para el aumento de la capacidad aeróbica (Chen et al., 2012). En este contexto, se ha propuesto que la suplementación permanente con ginseng podría mejorar la función cardiorrespiratoria y reducir la concentración de lactato en la sangre. Su efecto ergogénico es atribuido, principalmente, a sus compuestos bioactivos tales como ginsenósidos y ciwujianosides (Williams, 2006). También se ha recomendado este suplemento en casos de fatiga, para mejorar el acondicionamiento aeróbico, la fuerza y la recuperación post-ejercicio (Jin et al., 2020).

\section{Té verde (Camellia sinensis (L.) Kuntze)}

Pese a que en el último tiempo su uso se ha masificado como una manera de prevenir la ganancia de peso corporal, a nivel deportivo, la influencia de los extractos de té verde sobre el metabolismo no está del todo clara, existiendo resultados contradictorios en su uso durante el ejercicio (Senchina et al., 2011). Richards et al. (2010) evidenciaron que tras 48 horas de suplementación con 135 mg del principio activo epigalocatequin-3-galato del té verde, se producía una mejora en la capacidad aeróbica, aunque también se ha reportado una mejoría en la capacidad de resistencia cardiovascular, en el sistema de defensa antioxidante y en la oxidación lipídica (Sellami et al., 2018). Tsai et al. (2017), luego de 8 semanas de suplementación con extractos de té verde, en 8 jóvenes universitarios que realizaban actividad física regular al menos 3 veces por semana, determinaron que la suplementación no tuvo un efecto adicional en la síntesis de glucógeno muscular. No obstante, en los jóvenes suplementados, existió un aumento en la dependencia energética de la oxidación de grasas, en comparación con el grupo placebo (Tsai et al., 2017).

\section{Guaraná (Paullinia cupana Kunth)}

Posee un reconocido efecto sobre la activación del sistema nervioso central $y$, al igual que el té verde, se le ha atribuido un posible efecto sobre la reducción del peso corporal, posiblemente debido a su mayor contenido de cafeína (3,6\% a 5,8\%), en comparación a otras plantas como la del café (Coffea arabica L., 1\% a 2\%) (Sellami et al., 2018). De hecho, la suplementación por 8 semanas con una preparación herbal de Ma Huang (Ephedra sinica Stapf) y Guaraná, que contenía 72 mg/día de efedrina (principio activo del Ma Huang) y 240 mg/día de cafeína, redujo significativamente el peso corporal en adultos con sobrepeso (Boozer et al., 2001). Entre sus efectos no deseados se encuentran el insomnio, aumento de la ansiedad y malestar estomacal (Sellami et al., 2018). El efecto del Guaraná en la reducción del peso corporal podría ser considerada una ayuda en el deporte.

\section{Yerba mate (Ilex paraguariensis A.St.-Hil.)}

Es considerada una bebida estimulante, rica en antioxidantes y con un destacado rol en la oxidación de ácidos grasos (Matsumura et al., 2015). Se ha demostrado, además, que posee efectos sobre los niveles de colesterol, función hepática y diurética, y que podría aumentar el metabolismo lipídico durante el ejercicio (Godfrey et al., 2013). La ingesta de yerba mate (cápsula de 1000 mg) previo al ejercicio físico ha demostrado incrementar la oxidación de ácidos grasos y el gasto energético en individuos sanos (Areta et al., 2018). Otros investigadores han evidenciado que el consumo de infusiones de yerba mate sola (112 mg) o combinada con Guaraná o té verde, podría ser un coadyuvante en el tratamiento de la obesidad por su estimulación del catabolismo lipídico (Gambero \& Ribeiro, 2015). Adicionalmente, una de las características destacadas de la yerba mate es que, a diferencia de otros tés (como blanco o verde), no contiene catequinas, las cuales han sido vinculadas con toxicidad en el hígado (Heck \& de Mejia, 2007). El efecto de la yerba mate sobre el metabolismo lipídico, ha despertado también creciente interés científico en el ámbito de la medicina deportiva como potenciador del ejercicio y como una ayuda erogénica por sus efectos positivos en el rendimiento metabólico y físico (Areta et al., 2018).

\section{Jengibre (Zingiber officinale Roscoe)}

De manera histórica ha sido utilizado para combatir diferentes enfermedades, principalmente en culturas no occidentales. A nivel deportivo, su uso ( $2 \mathrm{~g}$ /día durante mínimo 5 días) ha sido documentado como un alternativo analgésico anti-inflamatorio (Wilson, 2015). Entre los posibles mecanismos, se ha propuesto que el jengibre bloquearía la actividad de las enzimas ciclooxigenasas (encargadas de la producción de prostaglandinas), así como 
también inhibiría la producción de citoquinas pro-inflamatorias en macrófagos in vitro (Tripathi et al., 2008). El ejercicio agudo y crónico induce la producción de citoquinas pro-inflamatorias, como la interleucina (IL)-8 y el factor de necrosis tumoral (TNF)-a, que no solo controla la inflamación inducida por el ejercicio, sino que también regula la disponibilidad de metabolitos para la contracción muscular (Capó et al., 2014). Sin embargo, pocos estudios han demostrado el efecto analgésico de esta raíz en deportistas (Sellami et al., 2018). El efecto del jengibre sobre la inflamación asociada al ejercicio podría considerarse una ayuda en el deporte.

\section{Ginkgo biloba L.}

Es principalmente utilizada para prevenir la pérdida de memoria, migrañas e incluso enfermedad de Alzheimer y Parkinson, por su efecto sobre la circulación (en especial circulación cerebral) y sus propiedades neuroprotectoras (reducción de formación del péptido $\beta$ amiloide y neurotoxicidad asociada) (Liao et al., 2020). A nivel deportivo, se ha visto que mejora el rendimiento en caminatas de corta distancia en pacientes con enfermedad arterial periférica (Schneider, 1992); sin embargo, en otros deportistas no ha presentado el mismo resultado (240 mg/día) (Wang et al., 2007), siendo considerada una planta segura en adultos sanos, pero en limitadas dosis (Sellami et al., 2018). Más estudios se necesitan para considerar a esta planta como una ayuda en el deporte.

\section{Conclusión}

Muchos son los suplementos deportivos, alimentos y plantas que se etiquetan como ergogénicos; sin embargo, son pocos los que realmente han demostrado su efectividad como tal. En la presente comunicación breve se han abordado algunos suplementos y plantas de uso frecuente que han evidenciado su efecto ergogénico y que además no han sido catalogadas como dopaje por las distintas federaciones internacionales (debido a su presencia en diversos alimentos habituales). No obstante, de todos los suplementos y plantas mencionados, se debe destacar que la cafeína si ha sido prohibida en competiciones deportivas en el pasado (Diel, 2020). Este alcaloide se encuentra de manera natural en el café, bayas de guaraná y hojas de té y yerba mate. A pesar de que estas hierbas pueden ejercer un efecto ergogénico en dosis adecuadas, las características individuales de tipo genéticas y metabólicas determinaran el efecto. Otras sustancias, como citrato de sodio, fosfatos y carnitina parecen tener potencial efecto ergogénico; sin embargo, la evidencia no es sólida. En este contexto, y considerando que la literatura es escasa o existe limitada evidencia de alta calidad en algunos de los estudios que se han llevado a cabo en la materia, es necesario profundizar la investigación en ayuda ergogénicas a través de estudios clínicos randomizados que permitan identificar una relación causa-efecto entre el suplemento o planta con potenciales características ergogénicas y el rendimiento deportivo. Esto permitirá realizar mejores análisis comparativos entre poblaciones, así como también entre el efecto que pudiesen poseer diferentes suplementos o plantas categorizadas como ergogénicas.

Por otro lado, es importante destacar que los ergogénicos descritos en esta comunicación reportan efectos, que, en su mayoría, son útiles en deportistas, en los que nuevos estímulos podrían marcar la diferencia entre ganar o perder una competición cuando los márgenes son estrechos. Una persona iniciada en el deporte, con una amplia posibilidad de mejoría en el rendimiento físico, difícilmente observará resultados en su rendimiento ya que la modesta ayuda ergogénica proporcionada por el suplemento pasa desapercibida en comparación al efecto del propio entrenamiento. Por lo tanto, el uso de estos suplementos y plantas debe plantearse sobre todo en el deportista bien entrenado que necesita de nuevos estímulos o nuevas estrategias para seguir aumentando el rendimiento deportivo.

Finalmente, es fundamental destacar que estos suplementos y plantas ergogénicas no deben recomendarse para obtener resultados ambiciosos en un corto período de tiempo.

\section{Contribución y reconocimientos: Fuentes de financiamiento}

El trabajo no tuvo financiamiento. Los autores declaran no tener conflictos de interés.

\section{Agradecimientos}

Este trabajo fue realizado en representación del consorcio de investigación ELHOC (Epidemiology of Lifestyle and Health Outcomes in Chile).

\section{Referencias}

Antonio J., Candow, D. G., Forbes, S. C., Gualano, B., Jagim, A. R., Kreider, R. B., ... Ziegenfuss, T. N. (2021). Common questions and misconceptions about creatine supplementation: what does the scientific evidence really show?. Journal of the International Society of Sports Nutrition 18, 13.

Areta, J. L., Austarheim, I., Wangensteen, H., \& Capelli, C. (2018). Metabolic and Performance Effects of Yerba Mate on Well-trained Cyclists. Med Sci Sports Exerc 50, 817-826.

Betteridge, S., Bescos, R., Martorell, M., Pons, A., Garnham, A. P., \& Stathis, C. C. (2016). No effect of acute beetroot juice ingestion on oxygen consumption, glucose kinetics, or skeletal muscle metabolism during submaximal exercise in males. J Appl Physiol 120, 391-398. 
Boozer, C. N., Nasser, J. A., Heymsfield, S. B., Wang, V., Chen, G., \& Solomon, J. L. (2001). An herbal supplement containing Ma Huang-Guarana for weight loss: a randomized, double-blind trial. Int J Obes Relat Metab Disord 25, 316-324.

Capó, X., Martorell, M., Llompart, I., Sureda, A., Tur, J. A., \& Pons, A. (2014). Docosahexanoic acid diet supplementation attenuates the peripheral mononuclear cell inflammatory response to exercise following LPS activation. Cytokine 69, 155-164.

Carr, A. J., Slater, G. J., Gore, C. J., Dawson, B., \& Burke, L. M. (2011). Effect of sodium bicarbonate on [HCO3-], $\mathrm{pH}$, and gastrointestinal symptoms. Int J Sport Nutr Exerc Metab 21, 189-194.

Chen, C. K., Muhamad, A. S., \& Ooi, F. K. (2012). Herbs in exercise and sports. Journal of physiological anthropology 31, 4-4.

Diel, P. (2020). Caffeine and Doping-What Have We Learned since 2004. Nutrients 12.

Gallardo Fuentes, F. J., Galloardo Fuentes, J. E., Ruiz Aguilar, C. A., Ramirez-Campillo, R., \& Rodriguez Rodriguez, F. J. (2015). Caracterización del consumo de suplementos nutricionales en población físicamente activa de gimnasios del sur de Chile. DIAETA, 33, 14-20.

Gambero, A., \& Ribeiro, M. L. (2015). The positive effects of yerba maté (Ilex paraguariensis) in obesity. Nutrients 7, 730-750.

Garthe, I., \& Maughan, R. J. (2018). Athletes and Supplements: Prevalence and Perspectives. Int J Sport Nutr Exerc Metab 28, 126-138.

Godfrey RJ, Laupheimer MW, S. S., \& a, e. (2013). A-Z of nutritional supplements: dietary supplements, sports nutrition foods and ergogenic aids for health and performance: Part 45. Br J Sports Med 47, 659-660.

Grgic, J., Rodriguez, R. F., Garofolini, A., Saunders, B., Bishop, D. J., Schoenfeld, B. J., \& Pedisic, Z. (2020). Effects of Sodium Bicarbonate Supplementation on Muscular Strength and Endurance: A Systematic Review and Meta-analysis. Sports Med 50, 1361-1375.

Guest, N. S., VanDusseldorp, T. A., Nelson, M. T., Grgic, J., Schoenfeld, B. J., Jenkins, N. D. M., ... Campbell, B. I. (2021). International society of sports nutrition position stand: caffeine and exercise performance. J Int Soc Sports Nutr 18, 1.

Hadzic, M., Eckstein, M. L., \& Schugardt, M. (2019). The Impact of Sodium Bicarbonate on Performance in Response to Exercise Duration in Athletes: A Systematic Review. Journal of sports science \& medicine 18, 271-281.
Heck, C. I., \& de Mejia, E. G. (2007). Yerba Mate Tea (Ilex paraguariensis): a comprehensive review on chemistry, health implications, and technological considerations. J Food Sci, 72, R138-151.

Hlinský, T., Kumstát, M., \& Vajda, P. (2020). Effects of Dietary Nitrates on Time Trial Performance in Athletes with Different Training Status: Systematic Review. Nutrients, 12.

Hord, N. G., Tang, Y., \& Bryan, N. S. (2009). Food sources of nitrates and nitrites: the physiologic context for potential health benefits. Am J Clin Nutr 90, 1-10.

Huerta Ojeda, Á., Tapia Cerda, C., Poblete Salvatierra, M. F., Barahona-Fuentes, G., \& Jorquera Aguilera, C. (2020). Effects of Beta-Alanine Supplementation on Physical Performance in Aerobic-Anaerobic Transition Zones: A Systematic Review and Meta-Analysis. Nutrients 12, 2490.

Jin, T.-Y., Rong, P.-Q., Liang, H.-Y., Zhang, P.-P., Zheng, G.-Q., \& Lin, Y. (2020). Clinical and Preclinical Systematic Review of Panax ginseng C. A. Mey and Its Compounds for Fatigue. Frontiers in pharmacology 11, 1031-1031.

Jorquera Aguilera, C., Rodríguez-Rodríguez, F., Torrealba Vieira, M. I., Campos Serrano, J., \& Gracia Leiva, N. (2016). Consumo, características y perfil del consumidor de suplementos nutricionales en gimnasios de Santiago de Chile. Revista Andaluza de Medicina del Deporte 9, 99-104.

Kreider, R. B., Kalman, D. S., Antonio, J., Ziegenfuss, T. N., Wildman, R., Collins, R., . . Lopez, H. L. (2017). International Society of Sports Nutrition position stand: safety and efficacy of creatine supplementation in exercise, sport, and medicine. J Int Soc Sports Nutr 14, 18.

Liao, Z., Cheng, L., Li, X., Zhang, M., Wang, S., \& Huo, R. (2020). Meta-analysis of Ginkgo biloba Preparation for the Treatment of Alzheimer's Disease. Clinical Neuropharmacology 43, 93-99.

Matsumura, M. D., Zavorsky, G. S., \& Smoliga, J. M. (2015). The Effects of Pre-Exercise Ginger Supplementation on Muscle Damage and Delayed Onset Muscle Soreness. Phytother Res 29, 887-893.

NHS. (2018). Bodybuilding and sports supplements: the facts. National Health Service (NHS) Report. Accedido en: https://www.nhs. uk/live-well/healthy-body/body-building-sports-supplements-facts/?tabname=seasonal-health. el 3 de Junio 2021.

Richards, J. C., Lonac, M. C., Johnson, T. K., Schweder, M. M., \& Bell, C. (2010). Epigallocatechin-3-gallate increases maximal oxygen uptake in adult humans. Med Sci Sports Exerc 42, 739-744. 
Santesteban Moriones, V. \& Ibáñez Santos, J. (2017). Ayudas ergogénicas en el deporte. Nutrición Hospitalaria 34, 204-215.

Saunders, B., Elliott-Sale, K., Artioli, G. G., Swinton, P. A., Dolan, E., Roschel, H., ... Gualano, B. (2017). beta-alanine supplementation to improve exercise capacity and performance: a systematic review and meta-analysis. Br J Sports Med 51, 658-669.

Schneider, B. (1992). [Ginkgo biloba extract in peripheral arterial diseases. Meta-analysis of controlled clinical studies]. Arzneimittelforschung 42, 428-436.

Sellami, M., Slimeni, O., Pokrywka, A., Kuvacic, G., L, D. H., Milic, M., \& Padulo, J. (2018). Herbal medicine for sports: a review. J Int Soc Sports Nutr 15, 14.

Senchina, D. S., Bermon, S., Stear, S. J., Burke, L. M., \& Castell, L. M. (2011). BJSM reviews: A-Z of nutritional supplements: dietary supplements, sports nutrition foods and ergogenic aids for health and performance. Part 17. Br J Sports Med 45, 150-151.

Senefeld, J. W., Wiggins, C. C., Regimbal, R. J., Dominelli, P. B., Baker, S. E., \& Joyner, M. J. (2020). Ergogenic Effect of Nitrate Supplementation: A Systematic Review and Meta-analysis. Med Sci Sports Exerc 52, 2250-2261.

Siegler, J. C., Marshall, P. W., Bray, J., \& Towlson, C. (2012). Sodium bicarbonate supplementation and ingestion timing: does it matter? J Strength Cond Res 26, 1953-1958.
Southward, K., Rutherfurd-Markwick, K. J., \& Ali, A. (2018). Correction to: The Effect of Acute Caffeine Ingestion on Endurance Performance: A Systematic Review and Meta-Analysis. Sports Med 48, 2425-2441.

Tripathi, S., Bruch, D., \& Kittur, D. S. (2008). Ginger extract inhibits LPS induced macrophage activation and function. BMC Complement Altern Med 8, 1.

Tsai, T.-W., Chang, C.-C., Liao, S.-F., Liao, Y.-H., Hou, C.-W., Tsao, J.-P., $\&$ Cheng, I. S. (2017). Effect of green tea extract supplementation on glycogen replenishment in exercised human skeletal muscle. British Journal of Nutrition 117, 1343-1350.

Van De Walle, G. P., \& Vukovich, M. D. (2018). The Effect of Nitrate Supplementation on Exercise Tolerance and Performance: A Systematic Review and Meta-Analysis. J Strength Cond Res 32, 1796-1808.

Wang, J., Zhou, S., Bronks, R., Graham, J., \& Myers, S. (2007). Supervised exercise training combined with ginkgo biloba treatment for patients with peripheral arterial disease. Clin Rehabil 21, 579-586.

Williams, M. (2006). Dietary supplements and sports performance: herbals. J Int Soc Sports Nutr 3, 1-6.

Wilson, P. B. (2015). Ginger (Zingiber officinale) as an Analgesic and Ergogenic Aid in Sport: A Systemic Review. The Journal of Strength \& Conditioning Research 29, 2980-2995. 